\title{
Presencia de bacterias asociadas a nieve marina en arrecifes influenciados por escorrentía continental del Caribe colombiano: primer acercamiento
}

\author{
Hernán Alejandro Henao-Castro, ${ }^{1}$ Elvira M. Alvarado Ch., ${ }^{2}$ \\ Laura C. Rodríguez ${ }^{3}$ y Johanna Santamaría ${ }^{4}$
}

'Investigador independiente. henaocastro.alejandro@gmail.com

2Investigadora independiente. Profesora de cátedra Universidad Jorge Tadeo Lozano. ealvaradochacon@gmail.com

${ }^{3}$ Estudiante de pregrado de Biología Marina. laurac.rodriguezr@utadeo.edu.co

4Profesora titular, Universidad Jorge Tadeo Lozano. johanna.santamaria@utadeo.edu.co

\section{Resumen}

Parte del deterioro de los arrecifes de coral se atribuye al efecto del aporte de agua dulce cargada de nutrientes y sedimentos finos. Estas condiciones favorecen la formación de nieve marina lodosa que transporta gran carga de bacterias que pueden ser patógenos de corales. Por lo anterior, se estudió la presencia de bacterias asociadas a nieve marina lodosa en una zona arrecifal del Caribe colombiano en un gradiente de distancia a la desembocadura del canal del Dique (Cartagena). Por medio de técnicas moleculares PCR-DGGE, se registró baja riqueza, con bandas representativas de siete bacterias diferentes. El número de especies varió según el sitio, pero no hubo relación con la cercanía a la fuente de escorrentía. Como estudio pionero, sirve como punto de partida para estudios en arrecifes del Caribe colombiano impactados por escorrentía.

Palabras clave: nieve marina lodosa, arrecifes de coral, escorrentía continental, bacterias asociadas a partículas y agregados, DGGE.

Editor: Hernández- Fernández, J.

Citation: Henao, H., Alvarado, E., Rodríguez, L., Santamaría, J. (2015). Presencia de bacterias asociadas a nieve marina en arrecifes influenciados por escorrentía continental del Caribe colombiano: Primer acercamiento. Revista Mutis 5(1); pág. 14-20.

Received: March 2, 2015; Accepted: May 20, 2015; Published on line: June 30, 2015

Copyright: $\odot 2015$ Henao et al. This is an open-access article, which permits unrestricted use, distributions and reproduction in any medium, provided the original author and source are credited.

Competing Interests: The authors have no conflict of interest.
Bacteria associated with muddy marine snow in coral reefs influenced by terrestrial runoff in the Colombian caribbean: first approach.

\begin{abstract}
Part of the degradation of coral reefs has been attributed to the effect of freshwater inputs with nutrients and fine-grain sediments. These conditions favor the formation of muddy marine snow which transports great bacterial loads that can be pathogenic to corals. Therefore, the presence of bacteria associated to muddy marine snow in a reef zone of the Colombian Caribbean in a distance gradient to
\end{abstract}


the mouth of the Dique Channel (Cartagena), was done. Using molecular techniques like PCR-DGGE, low species richness was registered by means of seven phylogroups possibly representing seven different species. The number of species differed according to the site, but no relation with the proximity to source point of run-off was found. As a pioneer study, it serves as a starting point for studies on reefs exposed to run-off.

Keywords: muddy marine snow, coral reefs, terrestrial runoff, particle-attached and aggregates associated bacteria, DGGE.

\section{Introducción}

El estudio de los arrecifes de coral en Colombia es relativamente reciente, comprendiendo entre 4 y 5 décadas (Pizarro y Alvarado, 2011). A pesar de la gran importancia de estos ecosistemas a nivel global, regional y local, se sabe que más del $60 \%$ se encuentran en amenaza directa o inmediata (Burke et al., 2011). Actualmente se han identificado un sinnúmero de amenazas naturales y antropogénicas. Las primeras comprenden huracanes, cambio climático, acidificación oceánica y calentamiento global, y las segundas incluyen sobrepesca, contaminación, alteración de condiciones medioambientales y escorrentía continental, entre otras. Esta última es de un gran interés en Colombia ya que es donde se ha reportado una de las mayores tasas de deforestación a nivel mundial, en especial a lo largo de la cuenca del río Magdalena (Restrepo et al., 2006) y, por consiguiente, se presenta la mayor descarga de sedimentos en el mar Caribe (Restrepo y Kjerfve, 2000). El aporte de agua dulce no solo cambia la salinidad, sino también disminuye la transparencia y aumenta la concentración de nutrientes y de partículas en suspensión (Wolanski et al., 2004). Con el cambio de la calidad del agua, se generan respuestas biológicas de los organismos que habitan los ecosistemas costeros, incluyendo los microorganismos. Así, los arrecifes de coral pueden presentar alteraciones como pérdida de biodiversidad, cambios estructurales y proliferación de bacterias patógenas, entre otras, que conllevan a una disminución de la resiliencia arrecifal y un posible colapso del sistema (Fabricius, 2011).

Los arrecifes coralinos se caracterizan por presentar aguas pobres en nutrientes (Stoddar, 1969) pero con una gran variedad de materia suspendida como material calcáreo, partículas detríticas orgánicas y mucus secretado por el plancton, algas, bacterias, e incluso el mismo mucus de los corales (Fabricius y Wolanski, 2000). Estas partículas en suspensión se agregan rápidamente por floculación así como por uniones químicas entre los exopolímeros. Estos agregados se asemejan a copos de nieve y son llamados nieve marina (Wolanski et al., 2003). La nieve marina se considera vital para procesos de remineralización de nutrientes y actualmente es fundamental en el secuestro de carbono hacia las profundidades oceánicas, siendo relevante en temáticas de cambio climático y calentamiento global (Alldredge y Silver, 1988). Se encuentra tanto en aguas continentales (nieve de río y lagos) como en las zonas estuarinas, las costeras y las oceánicas. Debido a su gran abundancia en mares templados, ha sido ampliamente estudiada en estos ambientes donde se registran agregados que van de centímetros a metros de diámetro en diferentes estratos de profundidad (Alldredge et al., 1986). A pesar de su presencia cosmopolita y de su evidente importancia, es poco lo que se sabe de este compuesto en ambientes arrecifales, a tal punto, que la mayoría de los estudios existentes se han realizado en la Gran Barrera de Coral australiana (Wolanski et al., 2003).

Si bien la nieve marina es vital en la dinámica de los ecosistemas marinos, a medida que se alteran las condiciones ambientales como temperatura, salinidad, concentraciones de nutrientes y material particulado en suspensión (p. ej. sedimentos finos), generalmente a través de escorrentía continental, este compuesto se transforma en nieve marina lodosa (Wolasnki et al., 2003) y puede tener efectos negativos como ahogamiento, abrasión y proliferación de organismos patógenos, letales para el sostenimiento de arrecifes coralinos. Estos efectos perturban el desarrollo y sobrevivencia de organismos arrecifales de la comunidad planctónica y bentónica, poniendo en riesgo la salud de los arrecifes coralinos (Fabricius y Wolasnki, 2000). Existen referentes de diversidad bacteriana en la columna de agua de arrecifes "prístinos" e impactados antropogénicamente (Dinsdale et al., 2008), que reflejan diferencias en la estructura y composición de las comunidades. En atolones del Pacífico Central, los arrecifes intervenidos antropogénicamente presentan hasta 10 veces más células microbianas y partículas semejantes a virus, que aquellas zonas no influenciadas por actividades humanas. 
Esta mayor abundancia es acompañada de una mayor fracción de bacterias heterótrofas, dominadas por un gran porcentaje de patógenos, lo cual está asociado a arrecifes con menor cobertura coralina y mayor incidencia de enfermedades en corales escleractínios (Dinsdale et al., 2008). Estudios experimentales han comprobado que la nieve marina enriquecida con nutrientes y lodos es más pegajosa, lo que dificulta su remoción en organismos filtradores (zooplancton) y sésiles (corales), resultando en mortalidad por ahogamiento (Fabricius y Wolanski, 2000). Asimismo, se ha determinado que este compuesto presenta mayor impacto en reclutas de corales duros, lo que implica afectaciones negativas en procesos de repoblamiento natural coralino (Fabricius et al., 2003).

En Colombia se conoce muy poco sobre la nieve marina. Solo se menciona dentro de estudios oceanográficos biológicos como componente importante del plancton, especialmente en el Pacífico (Betancur y Martínez, 2003; Giraldo et al., 2011). Aún más, no se ha estudiado en arrecifes coralinos ni se conocen las implicaciones de esta sobre los corales, especialmente en ambientes influenciados por escorrentía continental. Hasta la fecha, estudios como los de Bernal et al. (2006), Restrepo y Alvarado (2011) y Henao-Castro (2013), infieren que la nieve marina, especialmente la de tipo lodoso, podría estar presente y contribuyendo notablemente en el deterioro y degradación de arrecifes coralinos del Caribe colombiano, por lo cual recomiendan investigaciones en arrecifes degradados e influenciados por aportes continentales. Solo un estudio ha demostrado que existe una alta densidad de microorganismos heterótrofos asociados a partículas de nieve marina lodosa, principalmente cuando esta se encuentra en zonas con mayor influencia de escorrentía continental (Henao-Castro et al., 2015).

Los arrecifes del Archipiélago de Nuestra Señora del Rosario han exhibido un evidente deterioro en las últimas cuatro décadas, caracterizado por disminución de cobertura de corales e incremento de cobertura algal (Restrepo y Alvarado, 2011), así como un aumento en la incidencia de enfermedades coralinas (Navas-Camacho et al., 2010). Estos cambios se han atribuido a distintas fuentes de disturbio como calentamiento global, sobrepesca y especialmente a la escorrentía continental proveniente del canal del Dique (Alvarado y Corchuelo, 1992; Restre- po y Alvarado, 2011). Un estudio reciente en el Parque Nacional Natural Corales del Rosario y San Bernardo (Henao-Castro, 2013) registró aguas con alta concentración de nutrientes (nitratos y amonio $>27$ $\mu \mathrm{M})$ y sedimentos de tipo lodoso $(20,6 \%$ de la tasa de sedimentación total). Es posible, entonces, que la escorrentía continental en esta zona pueda estar promoviendo la formación de nieve marina lodosa con abundancia y diversidad de microorganismos, incluyendo patógenos para los corales. Por lo anterior, en esta investigación se realizaron los primeros acercamientos al estudio de la presencia de bacterias asociadas a nieve marina lodosa en zonas arrecifales de un área marina protegida del Caribe colombiano. Asimismo, se pretendió determinar diferencias en la riqueza de bacterias (asociadas a la nieve marina lodosa) a partir de un gradiente de distancia a la desembocadura del canal del Dique desde la bahía de Barbacoas.

\section{Métodos}

Para estudiar la riqueza de bacterias asociada a nieve marina se tomaron tres muestras $\left(\geq 1 \mathrm{~cm}^{3}\right)$ de flóculos presentes en la columna de agua entre los 8 y $10 \mathrm{~m}$ de profundidad en 6 sitios arrecifales del Archipiélago de Nuestra Señora del Rosario en un gradiente desde Isla Arena (cercano a la fuente de escorrentía) hasta Isla Tesoro (distante de la fuente). Cada muestra $(\mathrm{N}=18)$ se extrajo con jeringa estéril de $50 \mathrm{ml}$ mediante buceo autónomo durante 10 minutos. Por medio de técnicas moleculares (aislamiento de ADN total, reacción de amplificación en cadena del gen 16S rADN-PCR y gel de electroforesis de gradiente denaturante-DGGE) se obtuvo una imagen con diferentes patrones de bandas por cada sitio. En la reacción de amplificación de esta región se utilizaron los oligonucleótidos universales 341 F-GC (5'-CGC CCG CCG CGC GCG GCG GGC GGG GCG GGG GCA CGG GGG GCC TAC GGG AGG CAG CAG-3') y 907 R 5'-CCG TCA ATT CTT TTG AGT TT-3' que amplifican una región de 566 pb (Ishii and Fukui, 2001). La reacción de amplificación se llevó a cabo en un volumen final de reacción de $50 \mu \mathrm{L}$. El programa de gradiente de temperatura empleado para el termociclador marca Labmate fue: $94{ }^{\circ} \mathrm{C}$ por 4 minutos, seguido por 10 ciclos de $94{ }^{\circ} \mathrm{C}$ por 50 segundos, $59-49^{\circ} \mathrm{C}$ de temperatura de alineación por 45 segundos, un minuto de extensión a $72{ }^{\circ} \mathrm{C}$. Después, 18 ciclos de $94{ }^{\circ} \mathrm{C}$ por 50 segundos, $49{ }^{\circ} \mathrm{C}$ por 45 segundos, 1 minuto a $72{ }^{\circ} \mathrm{C}$ y un paso final de extensión a $72{ }^{\circ} \mathrm{C}$ por 7 minu- 
tos. El análisis de DGGE se llevó a cabo en un sistema Bio Rad Decode. Los productos de amplificación de PCR del gen 165 rADN, con los cuales se analizan las poblaciones de bacterias en las muestras, se corrió en un gel de poliacrilamida del $8 \%$ con un gradiente paralelo del $45 \%$ de urea formamida en la parte superior y de $50 \%$ en la base del gel. Este se corrió por 18 $\mathrm{h}$ a $40 \mathrm{~V} \mathrm{~cm}-1$ a $60{ }^{\circ} \mathrm{C}$ en buffer TAE $1 \mathrm{X}$. Para visualizar las bandas de DNA, el gel se tiñó en una solución de bromuro de etidio $(0.5 \mu \mathrm{g} / \mathrm{ml})$. Finalmente se tomó una fotografía de la imagen con el sistema fotodocumentador UVP Gel Doc-ittm 300. Cada banda representa una posible especie de bacteria, con lo que al final se obtuvo la riqueza taxonómica de cada muestra analizada. La extracción de ADN y reamplificación se realizó en los laboratorios de la Universidad Jorge Tadeo Lozano. La técnica de DGGE se llevó a cabo en el laboratorio de Biología Molecular Marina (BIOMMAR) de la Universidad de los Andes.

\section{Resultados Y Discusión}

Se detectaron siete filogrupos diferentes de bacterias asociadas a nieve marina lodosa en los arrecifes del Archipiélago de Nuestra Señora del Rosario, que oscilaron entre 1 y 4 por zona arrecifal (figura 1). Aunque no se han encontrado referentes en sistemas arrecifales coralinos, comparaciones en ambientes controlados en laboratorio (16 a 20 especies - Schäfer, 2002), en episodios de proliferación de microalgas en la costa de California durante la primavera cuando hay mayor disponibilidad de nutrientes (10 a 12 especies ? Fandino et al., 2001), y estudios en sistemas oceánicos del golfo de Trieste, mar Adriático influenciados por la presencia de ríos (más de 90 taxa - Rath et al., 1998), permiten inferir que el número de filogrupos presentes en los ambientes arrecifales del archipiélago es bajo. Es probable que la baja riqueza sea producto del tamaño de muestra pequeña $\left(1 \mathrm{~cm}^{3}\right.$ por muestra, 3 muestras por sitio). También podría ser causado por el uso de un gradiente o tiempo en el proceso de DGGE inadecuado, aunque para este estudio se utilizaron 13 gradientes con diferentes tiempos de corrido de geles, encontrandose que el gradiente de $45 \%$ de urea formamida en la parte superior y de $50 \%$ en la base del gel que corrió por $18 \mathrm{~h}$ a $40 \mathrm{~V}$ $\mathrm{cm}^{-1}$ a $60{ }^{\circ} \mathrm{C}$ permitió diferenciar las distintas bandas que representan a las especies de bacterias (figura 1). Por último, no se puede descartar que efectivamente la riqueza bacteriana asociada a nieve marina sea baja per se en el área de estudio, y por ausencia de referentes de investigaciones de diversidad bacteriana en nieve marina lodosa en arrecifes coralinos del mundo, no se tenga un referente para comparar o discutir el resultado. No obstante, de un estudio simultáneo en la zona de estudio se sabe que las densidades de bacterias asociadas a este tipo de nieve marina es alta en comparación con las abundancias de aquellas de vida libre e incluso de otras zonas arrecifales y estuarinas del Caribe (Henao-Castro et al., 2015). Así, existe la posibilidad de que haya baja riqueza con alta abundancia de esas pocas especies o filogrupos para el caso concreto del presente estudio.

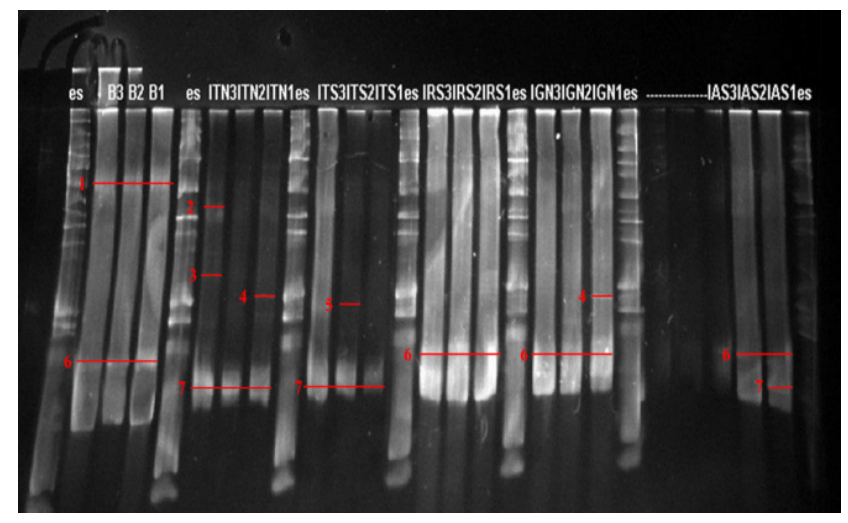

Figura 1. Electroforesis de gel de gradiente denaturante (DGGE) de nieve marina en zonas arrecifales del área marina protegida PNNCRy SB. Las muestras indican los arrecifes en un gradiente de lejanía a la fuente de escorrentía partiendo de la bahía de Barbacoas. En orden de lejanía, las estaciones de muestreo fueron Burbujas (B), Isla Tesoro Norte (ITN), Isla Tesoro Sur (ITS), Isla Rosario Sur (IRS), Isla Grande Norte (IGN) e Isla Arena Sur (IAS). En rojo se muestran las bandas que representan un posible filogrupo de los siete identificados.

Por otra parte, contrario a lo esperado, el número de filogrupos de bacterias no fue diferencial según el gradiente por aportes del canal del Dique (figura 2). Los patrones de banda, a partir de la comparación filogenética con la técnica DGGE, mostraron que Isla Tesoro Norte (sitio más distante a los aportes del canal del Dique) presentó la mayor riqueza $(S=4)$, mientras que Isla Rosario Sur, que también está alejado de la escorrentía del afluente continental, presentó el menor número de filogrupos $(\mathrm{S}=1)$. Las demás estaciones exhibieron dos filogrupos. Un patrón similar también se registró en bacterias adheridas a partículas en ambientes marinos estuarinos en el Puerto Victoria, Hong Kong (Zhang et al., 2007) donde no se encontraron diferencias en el número de especies ( $N=21$ - 24) en tres puntos con condiciones ambientales distintas 
como concentración de nutrientes y partículas en suspensión. Sin embargo, aunque la riqueza no mostró un patrón, sí se observan diferencias en los filogrupos presentes ya que algunos solo se presentaron en los sitios más alejados ( $\mathrm{Sp} 1$ en Burbujas y sp 2 y 3 en Tesoro Norte) mientras que la $\mathrm{Sp} 6$ se encontró en todos menos en las estaciones de Tesoro (figura 2).

Como estudio preliminar, el registro de bacterias diferentes asociadas a la nieve marina lodosa es un primer paso. Sin embargo, es necesaria la identificación de esas bacterias (filogrupos) a través de técnicas moleculares más avanzadas como pirosecuenciación para comprender el rol que tienen en los arrecifes de coral y si están asociadas a las enfermedades de los corales. Además es necesario cuantificar sus abundancias ya que las dominancias de las especies podrían dar indicios del impacto antropogénico en el sistema arrecifal de una forma diferencial, ya que se sabe que en ambientes intervenidos antropogénicamente, con elevadas concentraciones de nutrientes y sedimentos finos en suspensión, existe mayor número de bacterias heterótrofas (Henao-Castro et al., 2015) dentro de las cuales se podrían encontrar agentes patógenos para los corales (Dinsdale et al., 2008).

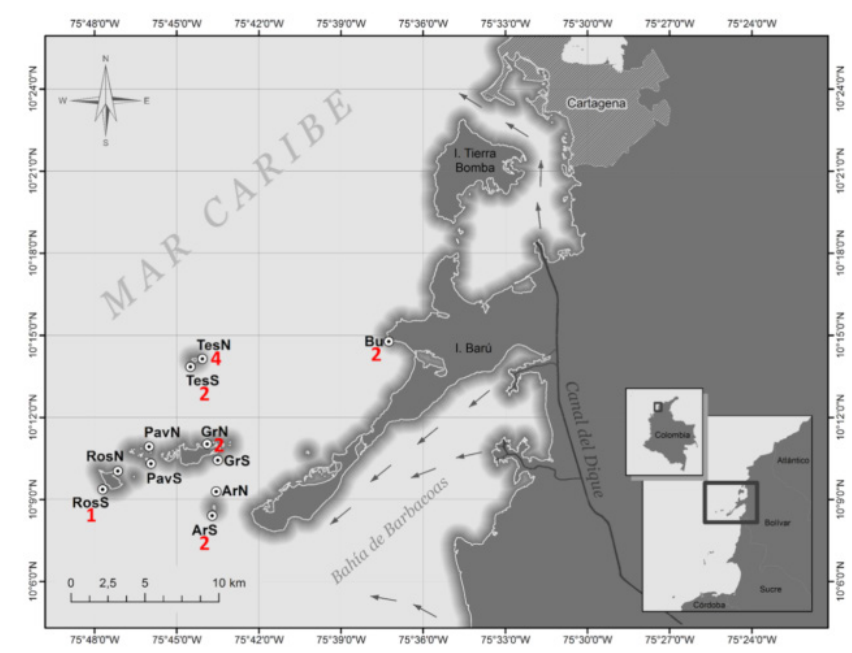

Figura 2. Número de filogrupos (color rojo) de bacterias asociadas a nieve marina lodosa en un gradiente espacial en zonas arrecifales del Archipiélago de Nuestra Señora del Rosario. Isla Arena Sur (ArS), Isla Arena Norte (ArN), Isla Grande Sur (GrS), Isla Grande Norte (GrN), Isla Pavitos Sur (PavS), Isla Pavitos Norte (PavN), Isla Rosario Norte (RosN), Isla Rosario Sur (RosS), Isla Tesoro Sur (TesS), Isla Tesoro Norte (TesN), Burbujas (Bu).

\section{Agradecimientos}

Los autores agradecen a la Universidad de Bogotá Jorge Tadeo Lozano por la financiación del proyecto de convocatoria interna № 8, 2011 "Nieve marina: biodiversidad de microorganismos en los arrecifes de coral del Archipiélago Nuestra Señora del Rosario, Área Marina Protegida". Al Capitán de Corbeta Carlos Andrés Martínez, a Esteban Zarza, Diego Duque y Ameth Vargas del Parque Nacional Natural Corales del Rosario y San Bernardo por el apoyo logístico y acompañamiento en la salida de campo para la colecta de muestras. Especial agradecimiento a Liliana López por su ayuda en los procesos de extracción y amplificación de ADN, así como en el protocolo de estandarización del DGGE. Al profesor Juan Armando Sánchez de la Universidad de los Andes por facilitar la infraestructura y reactivos del laboratorio BIOMMAR y a Mauricio Buitrago, de la misma Universidad por su asesoría y acompañamiento en el protocolo de DGGE. Finalmente, al profesor Javier Hernández de la Universidad Jorge Tadeo Lozano por facilitar la toma de imágenes con el fotodocumentador del laboratorio de Biología Molecular.

\section{Bibliografía}

Acinas, S. G., Antón, J., \& Rodríguez-Valera, F. (1999). Diversity of Free-Living and Attached Bacteria in Offshore Western Mediterranean Waters as Depicted by Analysis of Genes Encoding 16S Rrna. Appl. Environ. Microbiol. 65: 2514-522.

Alldredge, A. L., Cole, J. J.,\& Caron, D. A. (1986). Production of heterotrophic bacteria inhabiting macroscopic organic aggregates (marine snow) from surface waters. Limnol. Oceanogr. 31: 68-78.

Alldredge, A. L. \& Silver, M. W. (1988). Characteristics, dynamics and significance of marine snow. Prog. Oceanogr. 20: 41-82.

Alvarado, E. M., \& Corchuelo, M. C. (1992). Los nutrientes, la temperatura y la salinidad provenientes del Canal del Dique como factores de deterioro en el Parque Nacional Natural Corales del Rosario. En: VII Seminario Nacional de Ciencias y Tecnologías del Mar. Santa Marta. 277-287 p.

Bernal, G., A. Velásquez, I. Vargas, A. Agudelo, C. Andrade, J. Domínguez, C. Ricaurte, \& G. Mayo. (2006). Variabilidad de los aportes a los sedimen- 
tos superficiales durante un ciclo anual de los bancos de Salmedina. Bol. Invest. Mar. Cost. 35: 59-75.

Betancur, M., \& Martínez, I. (2003). Foraminíferos bentónicos recientes en sedimentos de fondo de la cuenca de Panamá (Pacífico colombiano), como indicadores de productividad y oxigenación. Bol. Invest. Mar. Cost.32: 93-123.

Burke, L., Reytar, K., Spalding, M., \& Perry, A. (2011). Reefs at risk revisited. World Resources Institute: Washington, DC. $130 \mathrm{p}$.

DeLong, E. F., Franks, D. G., \& Alldredge, A. L.(1993). Phylogenetic diversity of aggregate-attached vs. free-living marine bacterial assemblages. Limnol. Oceanogr. 38: 924-934.

Dinsdale, E., Pantos, O., Smriga, S., Edwards, R., \& Angly, F. (2008). Microbial ecology of four coral atolls in the Northern Line Islands. Plos One 3: 1-17.

Fabricius, K. (2011). Factors determining the resilience of coral reefs to eutrophication: a review and conceptual model. En. Dubinsky, Z. y N. Stambler. (Eds.). Coral reefs: an ecosystem in transition. Springer, $525 \mathrm{p}$.

Fabricius, K. E., \& Wolanski, E. (2000). Rapid smothering of coral reef organisms by muddy marine snow. Est. Coast. Shelf Sci. 50: 115-120.

Fabricius, K., Wild, C., Wolanski, E., \& Abele, D. (2003). Effects of transparent exopolymer particles and muddy terrigenous sediments on the survival of hard coral recruits. Est. Coast. Shelf Sci. 56: 1-9.

Fandino, L. B., Riemann, L., Steward, G. F., Long, R. A., \& Azam, F. (2001). Variations in bacterial community structure during a dinoflagellate Bloom analyzed by DGGE and 16S rDNA sequencing. Aquat. Microb. Ecol. 23: 119-130.

Giraldo, A., Valencia, B., \& Ramírez, D. (2011). Productividad planctónica y condiciones oceanográficas locales en Isla Gorgona durante julio 2006. Bol. Invest. Mar. Cost. 40:185-201.

Henao-Castro, H. A. (2013). Efectos de los aportes del canal del dique sobre el reclutamiento de especies de coral en los arrecifes del archipiélago Nuestra Señora del Rosario, área marina protegida. Te- sis de Maestría. Facultad de Ciencias Naturales e Ingeniería, Universidad de Bogotá Jorge Tadeo Lozano, Colombia.

Henao-Castro, H. A., Comba, N., Alvarado, E. M., \& Santamaría, J. (2014). Bacterias autótrofas y heterótrofas asociadas a nieve marina lodosa en arrecifes con escorrentía continental. Univ. Sci. 20: 9-16. DOI: 10.11144/Javeriana.SC20-1.baha

Ishii, K., \& Fukui, M. (2001) Optimization of annealing temperature to reduce Bbias caused by a primer mismatch in multitemplate PCR. Appl. Environ.Microbiol. 67: 3753-3755 DOI 10.1128/ AEM.67.8.3753-375567.

Kiorboe, T. (2001). Formation and fate of marine snow: small-scale processes with large-scale implications. Sci. Mar. 652: 57-71.

Navas-Camacho, R., Gil-Agudelo, D., Rodríguez-Ramírez, A., Reyes-Nidia, M., \& Garzón-Ferreira, J. (2010). Coral diseases and bleaching on Colombian Caribbean coral reefs. I. J. Trop. Biol. 58: 95-106.

Pizarro, V., \& Alvarado, E. M. (2011). Cambios que no hemos interiorizado: los arrecifes que aún desconocemos. Expeditio. 5: 63-69.

Rath, J., Wu, K., Herndl, G., \& DeLong, E. (1998). High phylogenetic diversity in marine-snow associated bacterial assemblage. Aquat. Microb. Ecol. 14: 261-269.

Restrepo, J., \& Alvarado, E. M. (2011). Assessing major environmental issues in the Caribbean and Pacific coasts of Colombia, South America: an overview of fluvial fluxes, coral reef degradation, and mangrove ecosystems impacted by river diversion. En: Wolanski E, McLusky D (Eds.) Treatise on Estuarine and Coastal Science. Waltham: Academic Press. DOI: 10.1016/B978-0-12-374711-2.01117-7.

Restrepo, J. D., \& Kjerfve, B. (2000). Magdalena River: interannual variability (1975-1995) and revised water discharge and sediment load estimates. J. Hydrol. 235: 137-149.

Restrepo, J. D., Zapata, P., Díaz, J. M., Garzón-Ferreira, J., \& García, C. (2006). Fluvial fluxes into the Caribbean Sea and their impact on coastal eco- 
systems: The Magdalena River, Colombia. Glob. Planet. Change. 50: 33-49.

Schäfer, H., Abbas, B., Witte, H., \& Muyzer, G. (2002). Genetic diversity of "satellite" bacteria present in cultures of marine diatoms. FEMS Microbiol. Ecol 42: 25-35.

Stoddart, D. (1969). Ecology and morphology of recent coral reefs. Biol. Rev. Cambridge Phil. Soc. 44: 433-498.

Wolanski, E., Richmond, R., McCook, L., \& Sweatman, H. (2003). Mud, Marine Snow and Coral Reefs: The survival of coral reefs requires integrated water- shed-based management activities and marine conservation. Amer. Sci. 91: 44-51.

Wolanski, E., Richmond, R., \& McCook, L. (2004). A model of the effects of land-based, human activities on the health of coral reefs in the Great Barrier Reef and in Fouha Bay, Guam, Micronesia. J. Mar. Sys. 46: 133-144.

Zhang, R., Liu, B., Lau, S., Ki1, J., \& Qian, P. (2007). Particle-attached and free-living bacterial communities in a contrasting marine environment: Victoria Harbor, Hong Kong. FEMS Microbiol. Ecol. 61: 496-508. 